\title{
Funktion und gattungsgeschichtliche Bedeutung des manichäischen Gigantenbuchs
}

\author{
JENS WILKENS (Göttingen)
}

\begin{abstract}
Du temps que la Nature en sa verve puissante Concevait chaque jour des enfants monstrueux, J'eusse aimé vivre auprès d'une jeune géante, Comme aux pieds d'une reine un chat voluptueux.

(Charles Baudelaire)
\end{abstract}

I.

Auch wenn wir das mythologische System Manis aus den manichäischen Originalquellen und den Sekundärberichten mittlerweile sehr gut kennen, so ist es um Manis eigene Werke doch eher schlecht bestellt. Meist verfügen wir lediglich über einzelne Blätter und Fragmente oder Zitate in manichäischen Originalwerken wie dem Kölner Mani Kodex, dem Synaxeis-Kodex oder in antimanichäischen Schriften. ${ }^{1}$ Die Listen seiner kanonischen Werke, die uns bekannt sind, ${ }^{2}$ differieren in dem einen oder anderen Punkt, aber einen mehr oder weniger geschlossenen manichäischen Kanon hat es sicherlich gegeben. Mani wollte bekanntlich die Verfälschung seiner Lehre dadurch verhindern, dass er seine Werke kodifizieren und gezielt vervielfältigen ließ.

Der vorliegende Beitrag ist einem Problem gewidmet, das der Forschung lange Zeit Rätsel aufgegeben hat, nämlich warum Mani ein zu seiner Zeit

1 Etwas besser sieht es beim Šābuhragān aus, dem einzigen Werk, welches Mani auf Mittelpersisch verfasste und das interessanterweise meist nicht in kanonischen Listen auftaucht, sowie bei den Briefen. Zum Šăbuhragān vgl. MACKenZIE 1979, 1980 und HUTTER 1992. In der letzten Zeit ist ein beträchtlicher Wissenszuwachs hinsichtlich Manis Lebendigen Evangeliums zu verzeichnen, von dem Zitate im koptischen SynaxeisKodex bewahrt sind. Vgl. FUnK 2009. Zu Manis Psalmen siehe DURKINMEISTERERNST/MORANO 2010.

2 Eine Zusammenstellung der Listen von meist sieben kanonischen Schriften Manis aus der manichäischen Originalliteratur und der häresiologisch-kritischen Auseinandersetzung mit seiner Lehre bietet REEVES (1992, S. 10-19). Vgl. auch MORANO 2008, S. 71-72 und GARDNER/LIEU 2004, S. 151-175 (mit Texten in Übersetzung). Zu Fragmenten kanonischer Werke Manis in der sogdischen Literatur vgl. die Zusammenstellung bei RECK 2006, S. 332. 
bekanntes apokalyptisches ${ }^{3}$ Werk des Judentums ${ }^{4}$ aus der Periode des Zweiten Tempels, ${ }^{5}$ das Gigantenbuch, umgearbeitet und seinem eigenen Schriftenkanon zugefügt hat. Mit der Umarbeitung des Gigantenbuchs und dessen Einbindung in seinen eigenen Kanon hat Mani das Werk nachweislich in einen anderen Kontext überfürt. ${ }^{6}$ Mit der Adaption ist in gattungsgeschichtlicher Hinsicht auch ein Genrewechsel verbunden, wie noch auszufuihren ist.

Keine Einigkeit herrscht hinsichtlich der Frage, ob Mani das aramäische Gigantenbuch als Grundlage seines eigenen Werkes verwendet hat oder eine nicht bezeugte - gnostische Version. ${ }^{7}$ Von Bedeutung ist in diesem Zusammenhang auch, wie sich der Religionsstifter gegenüber dem Judentum positioniert hat. Kürzlich wurde darauf hingewiesen, dass Mani vermutlich ein differenziertes Bild des spätantiken Judentums gehabt und dieses nicht nur ex negativo betrachtet habe, ${ }^{8}$ wie man früher meist annahm. Manis Haltung gegenüber dem spätantiken Judentum gehört zu den vielleicht komplexesten Problemen der Manichäismus-Forschung. Die Zuweisung spezifischer „Textgemeinschaften“ zu gewissen Textgruppen der Qumran-Literatur, in denen das aramäische Gigantenbuch überliefert ist, ist an sich schon umstritten. Auch das Postulat eines „henochischen Judentums“ wird nicht von allen Experten geteilt. ${ }^{9}$ Eine weitere Schwierigkeit ist dadurch gegeben, dass sich die vermeintlichen Spuren einer solchen Gruppierung, wenn es sie denn je gegeben hat, in der Zeit nach $70 \mathrm{n}$. Chr. verlieren. Die Traditionsstränge religionsgeschichtlich zu verfolgen scheint bislang kaum möglich. Kontakte Manis zu

3 Es gibt keine allgemein akzeptierte Definition des Begriffs „Apokalyptik“. Einen sehr guten forschungsgeschichtlichen Überblick bietet BEDENBENDER 2000, S. 32-61. Auch die religionsgeschichtlichen Hintergründe sind in der Forschung umstritten. So nehmen einige Autoren - etwa Kvanvig (1988) - einen mesopotamischen Hintergrund gerade für die Henochliteratur an.

4 VANDERKAM (2007, S. 16) weist darauf hin, dass in letzter Zeit häufiger der Terminus "Judentümer" (,Judaisms“) in der Zeit des Zweiten Tempels in der Forschung gebraucht werde. Vgl. ähnlich auch JACKSON 2004, S. 15.

5 Zur Datierung des aramäischen Gigantenbuchs (1. Hälfte des 2. Jh. v. Chr.) siehe PUECH 2000, S. 11.

6 In etwa vergleichbar ist der Vorgang mit der Übernahme des Henochbuchs im Christentum, zunächst in einer griechischen, später dann in einer äthiopischen (1 Henoch) und einer slavischen Fassung (2 Henoch). Vgl. KNIBB 2007, S. 20. Zu den griechischen Textzeugen vgl. op. cit., S. 33 und STUCKENBRUCK 2007, S. 42 f.

7 Vgl. die von SundermanN (1994a, S. 40 [697] f.) zusammengetragenen Positionen. Interessant ist in diesem Zusammenhang das Fehlen von Bezügen zu Henoch und den Wächterengeln in den gnostischen Texten von Nag Hammadi. Siehe hierzu REED 2005, S. 276. Zu den gnostischen Texten, die den Fall der Wächterengel behandeln, s. BAUCKHAM 1985, S. 321.

8 FUNK 2009, S. $122 \mathrm{ff}$.

9 Vgl. die ausführliche Kritik in Heger 2010, die sich gegen die Thesen von BoccACCini, Nickelsburg, Bedenbender und Collins (v. a. Collins 1998 und 2007) wendet. 
bestimmten jüdischen Traditionsgemeinschaften sind jedenfalls nicht auszuschließen. Die henochische mythische Tradition scheint nach der Katastrophe von $70 \mathrm{n}$. Chr. hauptsächlich in christlichen Kreisen fortzuleben. ${ }^{10}$ Gerade der Mythos vom Fall der Engel war in den christlichen Gemeinden des 2. Jh. weit verbreitet, ${ }^{11}$ so dass auch diese als mögliche Vermittler grundsätzlich in Frage kommen. Vorherrschende Meinung in der Forschung ist aber, dass Mani das Gigantenbuch bei den Elkesaiten kennengelernt habe. ${ }^{12}$

Aber welche Bedeutung hatte der in etwas anderer Ausprägung auch in Genesis 6:1-4 $4^{13}$ fassbare mythologische Stoff für Mani und seine Lehre? ${ }^{14}$ Welche Anschlussstellen, aber auch Brüche gibt es hinsichtlich des manichäischen Kernmythos?

10 Vgl. Bauckham 1985, S. 316, Jackson 2004, S. 86, KnibB 2001 und Reed 2005, S. 147-189. Im frühen rabbinischen Judentum wurde die Henochliteratur hingegen nicht weiter tradiert.

11 Siehe GiUlEa 2007, S. 263 mit Belegen.

12 So etwa Reed 2005, S. 248; Reeves 1992, S. 208; Reeves 1996, S. 45-48; SunderMANN 1994a, S. 47 [704]; PUECH 2000, S. 25 Anm. 58.

13 Die Frage, in welchem Verhältnis Genesis 6:1-4 und das Wächterbuch zueinander stehen, ob überhaupt eine direkte Abhängigkeit in der einen oder der anderen Richtung besteht, kann als nicht gelöst betrachtet werden. Vgl. die Erörterungen in BEDENBENDER 2000, S. 191 f. (Anm. 147) und REeD 2005, S. 53-57. Auch im Buch der Jubiläen spielt der Stoff eine bedeutende Rolle (s. hierzu REED (2005), S. 86-95). Vgl. zur Sache auch den Sammelband, der von AufFarth und STUCKEnBRUCK (2004) ediert worden ist.

14 Während in der Henochliteratur im Allgemeinen den gefallenen Wächtern die Schuld für Sünde und Verderben gegeben wird, so in Genesis den Menschen. Vgl. NiCKELSBURG 1977, S. 386. Siehe zu Genesis 6:1-4 auch HeNDEL 1987 und ZiMmerMANN/ZIMMERMANN 1999 (mit reichhaltigen Literaturangaben in Anm. 2). Der letztgenannte Aufsatz arbeitet den Unterschied zur Henochtradition heraus, der darin bestehe, dass in Genesis 6:1-4 von Göttern oder Gottwesen und nicht von Engeln die Rede sei (op. cit., S. 329). Die Autoren vertreten die These, dass es sich um den Mythos von einer Heiligen Hochzeit handele. WiTAKOwSKI (2009) sieht den Hauptunterschied beider Überlieferungen darin, dass in Genesis von einem Abstieg der Söhne Gottes (= der Engel) die Rede sei, während im Buch Henoch der Fall der Engel tatsächlich eine Rebellion gegen Gott darstelle. ReEves (1992, S. 185-187) unterscheidet drei jüdische und christliche Traditionen: 1. Die Tradition, welche die Wächter im Himmel für die Verfehlung verantwortlich macht und durch 1 Henoch 6, 1 Henoch 8688 und 1 Henoch 106-107 repräsentiert wird; 2. Die Tradition, die den Ursprung der Sünde nicht im Himmel lokalisiert, da die Wächter von Gott geschickt wurden, um die Menschen zu unterweisen. Erst auf der Erde seien sie durch die Schönheit der Frauen verführt worden. Eine solche Interpretation sei im Buch der Jubiläen, in den clementinischen Homilien, in der mittelalterlichen jüdischen und islamischen Folklore sowie eventuell im Gigantenbuch aus Qumran zu finden; 3. Die Tradition, welche die Wächterengel zu menschlichen Nachkommen Seths macht. Dieser Typus sei v. a. in christlichen Werken nachweisbar. 
II.

Um sich diesen Fragen anzunähern, ist es notwendig, kurz die wichtigsten Etappen in der Erforschung des manichäischen Gigantenbuchs zu skizzieren. Bis zum Jahr 1934 waren noch keine Originalfragmente bekannt, und die Vermutungen bezüglich der Inhalte des Werkes waren eher vage. In diesem Jahr veröffentlichte W. B. HENNING seinen Aufsatz „Ein manichäisches Henochbuch“, in dem er ein mittelpersisches Fragment edierte und zeigen konnte, dass es seinem Inhalt nach zur Henochliteratur in manichäischem Gewand gehörte. ${ }^{15}$ In diesem Aufsatz formulierte HenNing bereits seine Hypothese, warum Mani diesen Stoff aufgegriffen habe:

„Es war das bei Mani so stark ausgeprägte Interesse für Mythen und Sagen aus grauer Vorzeit, was ihn zum Henochbuch führte; die Sage von den gefallenen Engeln und ihren Riesensöhnen durfte er sich nicht entgehen lassen. Mit dem übrigen Inhalt der von ihm gelesenen Schrift wird er es ebenso gehalten haben, wie er es stets bei der Lektüre von Offenbarungsschriften andrer Religionen gehalten hat: stimmte er einigermaßen zu seiner Lehre, so wurde er als willkommene Bestätigung akzeptiert; die Stellen, die sich mit ihr in keiner Weise vereinen ließen, wurden verworfen und als ,Fälschungen" deklariert. "“16

Nach den epochalen Erkenntnissen, die wiederum HENNING in seinem bekannten Aufsatz „The Book of Giants“ aus dem Jahr 1943 dargelegt hat, waren es vor allem die Forschungen von MILIK, die erstmalig nachgewiesen haben, dass unter den Funden vom Toten Meer ein Werk in mehreren Handschriften erhalten ist, das frappierende Parallelen zum manichäischen Gigantenbuch aufweist. ${ }^{17}$ MiLIK vertrat eine ähnliche These wie HenNing zur Adaption des Gigantenbuches durch Mani. Es seien sein narrativer Charme und die zugrunde-

15 Er wies bereits darauf hin, dass er weitere ,persisch[e] Bruchstück[e] aus Manis Gigantenbuch" zur Publikation vorbereite.

16 HeNNING 1934, S. 32 [346].

17 S. Milik 1971 und 1976. Bemerkenswert ist die Tatsache, dass ein Motiv, das auch im Wächterbuch von großer Bedeutung ist, im manichäischen Gigantenbuch bewahrt ist: die Wächterengel als Lehrmeister verbotener Künste. Vgl. die wichtigen Bemerkungen bei REED 2005, S. 6: „Moreover, even despite the popularity of the Book of the Watchers in the first centuries after its composition, the instruction motif is absent or suppressed in almost all Second Temple Jewish sources and in the NT." Zu diesem Motiv im manichäischen Gigantenbuch (Text H sogdisch) bzw. in einem koptischen Auszug aus diesem (38. Kephalaion, S. 92.24-31) siehe HenNing 1943-1946, S. 70 f. [133 f.] und MORANO 2008, S. 81 f., 84. Das Motiv der Wächterengel als Übermittler himmlischer Kenntnisse ist eng verbunden mit dem Motiv ihrer unzulässigen sexuellen Verbindung mit den Töchtern der Menschen. Siehe ReED 2005, S. 27-46. Christliche Autoren wie Tertullian, Clemens von Alexandrien, Hermias u.a. griffen das Motiv ebenfalls wieder auf. Vgl. ReED 2005, S. 174-184 und BAUCKHAM 1985. 
liegenden metaphysischen Wahrheiten gewesen, die Mani angezogen hätten. ${ }^{18}$ Hervorzuheben ist ferner MILIKs Beobachtung, dass die eigentliche manichäische Terminologie in Manis Werk vergleichsweise unauffällig zu Tage trete. ${ }^{19}$

Viele Publikationen sind danach erschienen, die immer mehr Details geklärt haben. Aus manichäologischer Sicht sind es vor allem zwei Aufsätze von WERNER SUNDERMANN, die erwähnt werden müssen. ${ }^{20}$ Auch einige weitere alttürkische Fragmente konnten identifiziert werden. ${ }^{21}$ An kürzlich erschienenen Arbeiten zum manichäischen Gigantenbuch ist insbesondere die italienische Übersetzung aus dem Jahr 2008 von ENRICO MORANO zu nennen, die auch eine - hypothetische - Ordnung aller Fragmente bietet, sowie die Edition eines neuen sogdisch-parthischen Gigantenbuchfragments in einem Aufsatz desselben Autors. ${ }^{22}$

III.

Trotz des Materialzuwachses seit HENNINGs Zeiten ist eine grundsätzliche Schwierigkeit geblieben: sowohl vom manichäischen Gigantenbuch in seinen verschiedenen Versionen (mittelpersisch, sogdisch, alttürkisch sowie parthische und koptische Auszüge) als auch vom aramäischen Gigantenbuch sind nur Fragmente bekannt, deren Abfolge noch viele Rätsel aufgibt. ${ }^{23}$ Außerdem sind letztere in den meisten Fällen so stark beschädigt, dass kaum eine vollständige Zeile erhalten ist. ${ }^{24}$ Verkompliziert wird die Lage dadurch, dass in die östliche Überlieferung - und auf diese müssen wir uns hauptsächlich stützen, sehen wir von inhaltlichen Bezügen zum Gigantenbuch in der koptischen Literatur ab, iranische mythologische Stoffe eingeflossen sind. ${ }^{25}$ Offenbar waren die $\mathrm{Ma}-$

18 „Épris par son charme narratif et ému par quelques vérités métaphysiques sousjacentes, Mani décida de lui faire place parmi ses propres productions littéraires." (MILIK 1971, S. 126)

19 MiLik 1971, S. 126 f.

20 Siehe SUNDERMANN 1984 und 1994a.

21 Vgl. WiLKENS 2000.

22 Vgl. MORANO 2009.

23 Vgl. STUCKENBRUCK 1997b mit einer Kritik älterer Rekonstruktionsversuche. Zu den Manuskripten siehe Puech 2000, S. 10 ff. Eine neue Edition dieser Fragmente bei STUCKENBRUCK 1997a. Zur Bestimmung der Abfolge der manichäischen Fragmente siehe den Beitrag von ENRICO MORANO in diesem Band.

$24 \mathrm{Zu}$ einer auf den neuesten Forschungsstand gebrachten Aufstellung der Manuskripte, die diesem Werk zuzuordnen sind, nebst Angabe der zugehörigen Fragmente vgl. STUCKenBRUCK 2007, S. 60 f. Dort findet sich auch eine ausführliche Angabe der Publikationsorte.

$25 \mathrm{Zu}$ Recht weist SkJÆRvø (1995, S. 220) darauf hin, dass es unklar sei, wann die iranischen Elemente in die Gigantenbuchüberlieferung aufgenommen wurden. Er wendet 
nichäer auf der Suche nach Parallelen, um unterschiedliche mythologische Systeme miteinander in Beziehung zu setzen und zu ihrem eigenen Metasystem zu verarbeiten, welches die religiöse Deutungshoheit beanspruchte. Die Verarbeitung speziell iranischer Mythologeme hat den Charakter des Werkes gewiss verändert. ${ }^{26}$ Ein Punkt sei aber betont: die Benennung einiger Giganten in der iranisch-manichäischen Überlieferung mit Namen von Helden der iranischen epischen Literatur wie Sām und Narimān könnte durch die spätantike jüdische Überlieferung angeregt sein, dass die Nachkommen der Söhne des Himmels und der Menschentöchter die Helden der Vorzeit gewesen sind. ${ }^{27}$ Dieser Anknüpfungspunkt gab den Manichäern die Gelegenheit, die Heldenepik in den Missionsgebieten des Ostens einer Neubewertung und damit gleichzeitig einer Kritik zu unterziehen. Die Heldentaten von Sām und Narimān und anderen wurden buchstäblich umgewertet. Sie waren nunmehr lediglich Ausdruck roher Gewalt der Giganten, die schließlich folgerichtig zu deren selbstverschuldetem Ende führen musste. ${ }^{28}$ Eine Umwertung epischer Traditionen war natürlich besonders im Kontext der Ostmission unabdingbar, für die das Gigantenbuch eine bedeutende Rolle spielte, wie wir wissen. ${ }^{29}$ Man wird nicht fehlgehen, wenn man annimmt, dass der Bezug auf bekannte epische Traditionen durch die neue Kontextualisierung nicht ohne Reibungen vonstatten ging. ${ }^{30}$

Nach meiner Auffassung müsste angesichts der Tatsache, dass ein kanonisches Werk Manis im Osten der Diaspora eine Fortschreibung dahingehend

sich gegen die Annahme, dass Mani selbst schon diese Anleihen gemacht haben könnte. Für eine spätere Entwicklung plädierte schon HenNING (1943-1946, S. 52 [115]).

26 Der Nachweis der unterschiedlichen Mythenkreise und die thematischen Bezüge sind in vorbildlicher Weise vor einigen Jahren von SKJÆRVØ (1995) herausgearbeitet worden.

27 Vgl. HANSON 1977, S. 210.

28 Eine solche Kritik epischer Traditionen ist schon für das aramäische Gigantenbuch gegeben, indem etwa Gilgameš, Utnapištim und Humbaba als Giganten auftreten. Auch Gilgameš' Herkunft war ja sowohl himmlischer als auch irdischer Natur (s. REEVES 1992, S. 120 f.). Bemerkenswert für das Gigantenbuch ist jedoch, dass Gilgameš und Hobabiš (= Humbaba) beide zu den Giganten gerechnet werden und somit einer Partei zugehören, während im Gilgameš-Epos beide als Antagonisten auftreten. Zu den Namen der beiden Gestalten im Gigantenbuch siehe STUCKENBRUCK 2003, S. 325332. Sehr interessant ist die von STUCKENBRUCK (op. cit., S. 325) vorgetragene These, dass auch Gilgameš' eitler Versuch, nach dem Tod seines Freundes Enkidu nach der Unsterblichkeit zu suchen, die Entwicklung seines Charakters im Gigantenbuch bestimmt haben könnte. Zu Utnapištim s. speziell auch REEVES 1993.

29 Vgl. die parth. Handschrift M 5815 (siehe BöHLIG/ASMUSSEN 1995, S. 100-102, hier: 101).

30 So sind z. B. Sāxm und Narīmān interessanterweise als Personennamen bei den Manichäern bezeugt. Vgl. SUNDERMANN 1994b, S. 254 f. [495 f.], 263 [504]. HUTTER (2004, S. 135a) hat Spuren einer historischen „Konkretisierung“ in der von HeNNING als Handschrift A des Gigantenbuchs gekennzeichneten Tradition entdeckt (M 101i) und diese Mani selbst zugeschrieben. 
erfahren hat, dass durch Einfügung neuer Namen auch Mythenkreise anderer religiöser Traditionen gleichsam attrahiert wurden, der Kanonbegriff spezifiziert werden. In den letzten Jahren haben sich viele Studien an JAN ASSMANNs bekannter Definition orientiert, die davon ausgeht, dass mit der Ausbildung eines Kanons dieser „,den Traditionsstrom ein für alle mal stillstellt““.31 Auch wenn im Falle des Manichäismus ein geschlossener Schriftenkanon postuliert werden kann, so gilt der Prozess der Schließung doch nicht für jedes einzelne Werk. ${ }^{32}$ Das Gigantenbuch war hinsichtlich seines Wortlautes nicht unantastbar, sondern konnte den Erfordernissen entsprechend umgearbeitet und den lokalen Gegebenheiten angepasst werden. Hierzu zählt auch die Einbindung des Sayan-Gebirges (kögmän) in die uigurische Fassung des Textes.

Hinsichtlich der Frage, warum Mani das Gigantenbuch umgearbeitet hat, darf selbstverständlich auch die Figur Henochs nicht vernachlässigt werden. Ist Henoch für Mani nur deshalb so wichtig, weil er mit dem Stoff des Gigantenbuchs in Verbindung steht, oder spielt er schon für sich genommen eine große Rolle? In der Abfolge der manichäischen Lichtapostel steht Henoch weit am Anfang als Patriarch aus der Zeit vor der Sintflut. Auch die visionäre Begabung Henochs und dessen Nähe zu Gott und damit seine Kenntnis kosmischer Geheimnisse sind $\mathrm{zu}$ berücksichtigen. Jedenfalls verkörpert Henoch offenbar in bestimmten Kreisen des spätantiken Judentums eine Art Gegenbild zu Moses. ${ }^{33}$ Trotz Henochs Bedeutung in der Sukzession der Lichtapostel können noch andere Faktoren benannt werden, die die Inkorporierung des Gigantenbuches in Manis Schriftenkanon angeregt haben mögen.

IV.

Die Beziehungen der manichäischen Literatur und der in ihr formulierten Inhalte zur Literatur des Judentums des Zweiten Tempels in Gestalt der Apokryphen und der Pseudepigraphen sind äußerst komplex. REEVES hat vermutet, dass die mit der Henochliteratur verbundenen Lehren eine deutlich größere Rolle in der Entstehung der manichäischen Lehre gespielt haben müssen, als man lange angenommen hatte. ${ }^{34}$ Die Bezüge zum Mythos vom Fall der Wächter sind aber in der ,,eigentlichen“ manichäischen Literatur wenig entwickelt. Es ist, um ein Beispiel zu geben, bemerkenswert, dass in den Primär-

31 ASSMANN 2002, S. 222.

32 Vgl. auch GARDNER/LIEU 2004, S. 151: „It is noticeable that, whilst Mani’s emphasis on the importance of his books is remarkable, there was some difficulty in maintaining closure to their number, order, and perhaps even contents."

33 Auch im frühen Christentum wird Henoch zuweilen als unbeschnittener ,"christlicher“ Prophet im Zeitalter vor der Sintflut porträtiert. Siehe REED 2005, S. 158 f. Zu Henoch im Manichäismus vgl. KLIMKEIT 1980.

34 Reeves 1991, S. 295. Kritisch zu ReEves etwa Hutter 2004, S. 134a. 
quellen und in sekundären antimanichäischen Berichten das Strafgericht an den Wächtern und den Giganten sowie die Sintflut, ${ }^{35}$ sieht man von wenigen Ausnahmen ab, keine Bedeutung haben. Diese Ereignisse sind für den Kern der manichäischen Lehre, die Befreiung der Lichtteilchen aus der Finsternis und die endgültige Trennung der beiden antagonistischen Prinzipien, wenn nicht irrelevant, so doch ein Nebenschauplatz. Eine urzeitliche Vernichtung des Menschengeschlechts durch die Sintflut mit Ausnahme von Noah und seinen Nachkommen liegt nach manichäischem Verständnis nicht im göttlichen Heilsplan, da dies zur Rettung der Lichtteilchen nichts beizutragen vermag. Das Gegenteil gilt für die Henochliteratur, und hier insbesondere für das Korpus, das in den Funden vom Toten Meer bewahrt ist: hier stellt der Fall der Engel den Zentralmythos, die Meistererzählung dar, die über Jahrhunderte die Entstehung neuer Werke anregen und nichts Geringeres als den Ursprung des Bösen in der Welt erklären sollte. ${ }^{36}$ Diese Erzählung, die letzten Endes ein Astralmythos ist, existiert in unterschiedlichen Varianten. ${ }^{37}$

In der ostmanichäischen Überlieferung gibt es terminologische Bezüge, die diesen adaptierten Mythos mit dem manichäischen Kernmythos verknüpfen. So bezeichnet z.B. der Terminus mäzendarān im Mittelpersischen sowohl die Wächter als auch die Giganten als auch die Archonten, die laut der manichäischen Kosmologie in den Himmeln gefesselt sind. ${ }^{38}$ Vermutlich wurde diese Terminologie bewusst gewählt, um eine Identifikation der henochischen und der manichäischen Mythenkreise zu erleichtern. ${ }^{39}$

35 Die Sintflut stellt im jüdischen Verständnis des Mythos eine bedeutende Zäsur dar. STUCKENBRUCK (1997b, S. 22) verweist aber mit Recht darauf, dass nur die fleischliche Existenz der Giganten zerstört worden sei, während ihre Geister überleben und die Menschen weiterhin plagen könnten.

36 Siehe Delcor 1976 [1979] und Nickelsburg 2001, S. 47. Zur Sonderstellung des aramäischen Gigantenbuchs innerhalb der Henochliteratur, v. a. was die Rolle Henochs angeht, vgl. STUCKENBRUCK 2003, S. 318 ff. Auch wurde darauf hingewiesen, dass eine Besonderheit dieses Werkes darin besteht, dass die Vorgänge aus der Perspektive der Giganten geschildert werden, die auch namentlich genannt werden. Vgl. REED 2005, S. 96.

37 Diese Varianten wurden vor einigen Jahren von JACKSON (2004) im Anschluss an die Terminologie von THOMAS KUHN als „Exemplare“ eines „henochischen Paradigmas“ bezeichnet. Einschlägige Stellen aus dem Wächterbuch, die den astralen Hintergrund des Mythos beleuchten, sind bei JACKSON 2004, S. 26, zusammengestellt.

38 Vgl. SundermanN 1994a, S. 42 [699] und Hutter 2004, S. 134b.

39 SundermanN (1994a, S. 43 [700]) führt noch den mittelpersischen Terminus dēwān an, der sowohl die Wächter als auch die Fehlgeburten in Manis System bezeichnen kann. SundermanN interpretiert den Vorgang hinsichtlich der Terminologie von māzendarān und dēwān folgendermaßen: „In both cases the fuzziness of the Manichaean terminology is best explained as the simple result of the straightforward and complete demonization of the fallen angels in a strictly dualistic doctrine. It concerns terms only; it does not indicate a conceptual fuzziness or a particular gnostic involvement." 


\section{V.}

Es gibt aber noch weitere Hinweise darauf, wie der Mythos vom Fall der Engel in das manichäische System inkorporiert wurde. Eine bekannte Passage stammt aus dem 38. Kapitel der Kephalaia ${ }^{40}$ :

„Wegen der Tücke und der Rebellion[,] die in der Wache des Großen Königs der

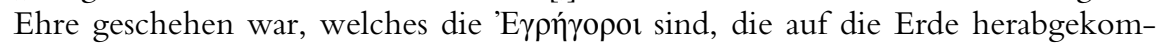
men waren aus den Himmeln, - ihretwegen wurde den vier Engeln Auftrag erteilt

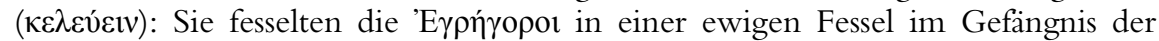
Finsteren (?), ihre Söhne wurden auf der Erde vernichtet. - Denn die Aborte waren ebenfalls im Wachbezirk des Adamas herabgekommen und hatten Adam und Eva geboren.“ (Kephalaia Kap. 38, S. 93.23-30)

Die Rebellion hat also nach der Entstehung des Kosmos stattgefunden, und zwar nachdem die Aufsicht über die verschiedenen Bezirke verteilt worden ist. Es hat nach diesem Kephalaion zwei Rebellionen gegeben: 1. die der Wächter; 2. die der Aborte. Die Egregoroi wurden bereits als Wesen der Finsternis der Aufsicht des Rex Honoris unterstellt, und ihnen gelang nur die Flucht. Weitere Stellen sowie die Bezeichnung dieser Wesen als dēwān in den mittelpersischen Fragmenten zeigen deutlich, dass sie nach manichäischem Verständnis bereits böse Wesen vor ihrer Rebellion gewesen sein müssen. Während die jüdische Tradition die Wächterengel als originär gute Wesen einstuft, sind sie im Manichäismus nur „entlaufene Strällinge“, die mit den am Firmament gekreuzigten Archonten bzw. mit einem Teil von ihnen identisch sind und der ihnen zugemessenen Bestrafung zeitweilig entkommen. ${ }^{41}$ Die Bestrafung, der die abgefallenen Wächter zugeführt werden, ist im 38. Kephalaion im manichäischen Kontext und in der jüdischen Henochliteratur jedoch ähnlich: Sie werden eingekerkert.

Die Entstehung der Giganten, der Söhne der Egregoroi, geht parallel mit der Entstehung des ersten Menschenpaares, Adams und Evas. Offenbar spielen, anders als in der jüdischen Version des Mythos von der Rebellion, die Töchter der Menschen bei der Entstehung der Giganten eine untergeordnete Rolle, da sie in den erhaltenen Fragmenten und Auszügen gar nicht ${ }^{42}$ oder nur en passant ${ }^{43}$ erwähnt werden. Das ist bemerkenswert, da die unerlaubte Verbindung der Menschentöchter mit den abgefallenen Engeln ein zentraler Punkt in der jüdischen Version des Mythos ist. Die Aborte sind hingegen sehr wohl ein

40 Alle Zitate aus den Kephalaia entstammen der Edition Polotsky/BÖHLIG 1940.

$41 \mathrm{Zu}$ dieser Interpretation vgl. ReEves 1992, S. 160 (Anm. 384). Zu den Archonten, die der Aufsicht des Königs der Ehre unterstellt sind, vgl. das 36. Kephalaion (S. 87.3088.33).

42 So in den Texten L, M, P (koptisch), J (mittelpersisch), K (sogdisch), Zu4 (uigurisch) und $\mathrm{H}$ (sogdisch). Vgl. MORANO 2008, S. 81-83, 84.

43 So in Text X (parthisch). Vgl. Morano 2008, S. 83. 
wichtiger Teil des manichäischen Kernmythos. ${ }^{44}$ Immerhin scheint die Entstehung des ersten Menschenpaares nach dem manichäischen Mythos eine Folge einer Art pervertierter Heiliger Hochzeit zu sein, die mit Kannibalismus verbunden ist. Ebenso ist in Genesis 6:1-4 und im Grunde auch in der Henochliteratur die Verbindung der Wächter mit den Töchtern der Menschen eine Form einer ins Negative verkehrten Heiligen Hochzeit, ${ }^{45}$ die schließlich zur Entstehung des kannibalisch veranlagten Gigantengeschlechtes führt. Werden in einem hieros gamos dynamische, schöpferische Kräfte freigesetzt, die für Fruchtbarkeit und Gedeihen sorgen, so kommt es im Mythos der gefallenen Wächter und im Manichäismus im Falle des Erzdämonenpaars zu einer Gegenschöpfung durch den deregulierten sexuellen Akt, wobei im Manichäismus letzten Endes ein Heilsplan dahinter steckt. ${ }^{46}$ Von hohem Interesse ist in diesem Zusammenhang der sogdische Text M 7800 /I/, der zum ersten Mal von SUNDERMANN ediert worden ist. ${ }^{47}$ Er handelt von den Fehlgeburten, die ihre Kinder dem Paar Šaqlūn und Pēsūs bringen, die sie verspeisen und die daraufhin kopulieren und so das erste Menschenpaar schaffen. Während M 7800 /II/ zum Gigantenbuch gehört, wurde M 7800 /I/ in HENNING (1943-1946) nicht berücksichtigt. Wie SUNDERMANN, so hält auch MORANO (2008, S. 105) eine Zugehörigkeit von M 7800 /I/ zum Gigantenbuch für denkbar:

„Tuttavia la storia degli aborti potrebbe avere preceduto o seguito quella dei Vigilanti e dei Giganti contenuta nell'altra pagina del bifolio, e l'appartenza dei due

$44 \mathrm{Zu}$ den Aborten in diesem Kontext vgl. Reeves 1992, S. $71 \mathrm{f}$. Es ist auch daran zu erinnern, dass offenbar Mani selbst die Nephilim (Riesen, Giganten) als 'Fehlgeburten' aufgefasst hat. Dieser Terminus sei für ihn identisch mit 'Tier' gewesen (HeNNING (1943-1946), S. 53 [116]). Zur etymologischen Verbindung der Bezeichnung Nephilim mit nēpel 'Fehlgeburt' vgl. COXON 1999, S. 619a-b. Siehe zu den Nephilim PERLITT (1990), S. 38-46, wo auch die Etymologie des Terminus Nephilim diskutiert wird (mit Hinweisen auf die Verbindung mit der Bedeutung 'Fehlgeburt' auch in der Forschungsliteratur). Lässt sich durch diese Identifikation auch die „Verdrängung“ der Giganten aus dem Kernmythos der Manichäer erklären? Der zitierte Passus aus den

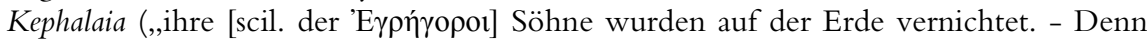
die Aborte waren ebenfalls im Wachbezirk des Adamas herabgekommen und hatten Adam und Eva geboren.") scheint diese Identifikation zu bestätigen.

45 ZimMERMANN/ZimMERMANN 1999. Siehe auch die unter Vorbehalt vorgetragene Hypothese in DELCOR 1976 [1979], S. 29 [289]: „La persistance dans le livre d'Hénoch de ces légendes sur l'union des anges liée au mont Hermon [...] pourraient être la survivance du hieros gamos accompli en ce haut lieu par quelque dieu cananéen."

46 Vgl. auch die treffenden Bemerkungen von ReEVES (1992, S. 74): „It will be noticed that, with one exception, the diet of the abortions agrees in every detail with that of the Enochic Giants. The exception is the consumption of human beings. Since, according to the Manichaean myth, humanity has yet to appear upon earth, the abortions cannot indulge in anthropophagy." Aber man kann hinzufügen, dass das Motiv des Kannibalismus beim Erzdämonenpaar, das die beiden ersten Menschen zeugt, gegeben ist. Dies vermerkt REEVES (1992, S. 197) an späterer Stelle.

47 Sundermann 1994a, S. 45 [702] f. Siehe auch Reeves 1996, S. 98 f. [Anm. 74]. 
fogli allo stesso libro induce a credere che anche questo frammento faccia parte del Libro dei Giganti.“

Die Wahrscheinlichkeit, dass diese Schlussfolgerung korrekt ist, erhöht sich durch die gemeinsame Erwähnung der Rebellion der Aborte und der Wächter in den Kephalaia. Der Mythos von der Rebellion der Wächter spielt im manichäischen Mythos nur eine untergeordnete Rolle und ist nicht mit der Funktion der Aborte gleichzusetzen. Gerade die Gegenüberstellung lässt die Rebellion der Wächter als peripher erscheinen. Noch weiter relativiert wird diese im 70. Kephalaion, wo es über die 'Lager', die den fünf Söhnen des Lebendigen Geistes unterstehen, heißt: 'Drangsal ( $\theta \lambda \hat{1} \psi(\varsigma)$ kam (auf) in diesen fünf Wachen von Zeit zu Zeit.' (S. 171.11-12).

Rebellionen sind folglich in allen fünf Wachbezirken, die den fünf Söhnen des Lebendigen Geistes unterstehen, immer wieder zu verzeichnen gewesen. Hier werden wiederum die Egregoroi der Wache des Königs der Ehre und die Aborte dem Bezirk des Lichtadamas zugewiesen. Der beispiellose, einmalige Akt der Rebellion gegen Gott in der jüdischen Tradition ist im Manichäismus eine immer wieder vorkommende Erschütterung, die tatsächlich auch wörtlich genommen werden kann, da diese Rebellionen auch mit Erdbeben in Verbindung gebracht werden.

\section{VI.}

Von herausragender Bedeutung, will man die Funktion des manichäischen Gigantenbuchs herausarbeiten, ist das Petersburger mittelpersische Doppelblatt S I O/120 (= L), das von WERNER SUNDERMANN im Jahr 1984 publiziert worden ist. Das erste Blatt enthält Teile des eigentlichen Gigantenbuchs, während das zweite dogmatischen Stoff bietet, der eine Art Vorstufe zu den Systematisierungen des Sermons vom Licht-Nous darstellt. ${ }^{48}$ Das Thema ist die Schaffung des Neuen Menschen aus dem Alten durch den Licht-Nous. SUNDERMANN hält es mit guten Gründen für möglich, dass dieses Überlieferungsgut auf altes Gedankengut Manis zurückgehen könnte und eventuell Teil des Gigantenbuches war. ${ }^{49}$ Diese Schlussfolgerung ist meiner Auffassung nach sogar unausweichlich, denn in der Überschrift des zweiten Teils steht wizārišn 'Deutung'; in Parabeln wird mit diesem Terminus z.B. das Epimythion bezeich-

48 Wieviel Text zwischen beiden Teilen ursprünglich anzusetzen ist, ist unklar. Der mittelpersische Text gehört nicht zum eigentlichen Sermon, ist aber mit diesem sehr eng verwandt. Der Sermon ist nicht auf Mittelpersisch überliefert.

49 „Es ist möglich, daß diese Überlieferung seit alters mit dem Gigantenbuch in engem Zusammenhang stand, ja vielleicht dieser Schrift Manis angehörte." (SUNDERMANN 1984, S. 502 [626]). 
net. ${ }^{50}$ Zehn Jahre später hat sich SUNDERMANN noch einmal kurz zu diesem Blatt geäußert und eine These formuliert, die zum ersten Mal die tatsächliche Funktion des manichäischen Gigantenbuchs erklären hilft. Diese These besagt, dass Gedankengut und Ethik des Gigantenbuchs und des 38. Kephalaions sehr eng zusammenhängen. Das Kephalaion beschreibt ein makrokosmisches Muster wiederkehrender Rebellionen, die ihre Parallele auf mikrokosmischer Ebene haben. Nach SUNDERMANN wird diese Entsprechung auch im zweiten Teil des Petersburger Gigantenbuchfragments S I O/120 (= L) zum Ausdruck gebracht. ${ }^{51}$ Die Geschehnisse des Gigantenbuchs werden durch die Lehren des traktatähnlichen Textes der „Deutung“ im Petersburger Fragment erklärt, insbesondere die Rebellion. Noch im Sermon vom Licht-Nous spricht man ja von den Dämonen, die gegen den Neuen Menschen kämpfen. Es handelt sich um eine Art mikrokosmischer Rebellion.

In der „Deutung“ des Petersburger Gigantenbuchtexts und im Sermon vom Licht-Nous wird mehrfach von der Fesselung und Einkerkerung gesprochen. Es geht um eine Transformation, eine Heilwerdung und Heiligung, die im Sermon durch den Licht-Nous bewirkt wird, wie im Wächterbuch (1 Henoch 1-36) die Erde durch das Eingreifen der Engel von den Giganten befreit und wieder geheilt werden soll. ${ }^{52}$ In der Henochliteratur und speziell im Wächterbuch und im Gigantenbuch soll dem Blutvergießen, das durch die Giganten heraufbeschworen wurde, ein Ende bereitet werden. Hiermit ist auch die Sintfluterzäh-

50 Nach SundermanN 1984, S. 498 [622], könnte der Terminus hier '(dogmatische) Belehrung' bedeuten. Da aber beide Teile des Doppelbuchblattes in enger Beziehung stehen, ist für wizārišn die Grundbedeutung 'Deutung' wahrscheinlicher.

51 „The tentative explanation I have been envisaging for some time presupposes that the 'Book of the Giants' follows the same line of thought and advocates the same morals as the 38th Coptic Kephalaion 'On the Light Nous, the apostles and the saints.' The outstanding topic of this long discourse is the permanent endangering of the perfectness of the New Man by the demoniac powers of flesh imprisoned in the bodies of the perfect. It is one of the achievements of the Light Nous to subdue and defeat the attacks of the Old Man. The temptations and vexations of the Old Man have in the Coptic text a cosmological, as it were macrocosmic pattern: the recurring rebellions of the demons in the skies against their divine overseers, the Sons of the Living Spirit. [...] The following phrase 'So ist es auch mit dem Körper' forms the transition to the anthropological interpretation and ethical evaluation of the old mythological tales." (SUNDERMANN 1994a, S. 48 [705]). Und weiter: „The vexing experience that the powers of darkness keep rebelling against the New Man must have been of great concern for everyday life in Manichaean communities. [...] That it does have its place in Mani's 'Book of the Giants' itself is made very likely by the second half of the St. Petersburg fragment." (loc. cit.). Bestätigung erfahren SUNDERMANNs Ansichten dadurch, dass ähnliche Gedanken schon im Wächterbuch als Subtext angelegt sind: „The Watcher Story as a whole contains three basic themes or codes [...]: The code of forbidden sexuality, the code of forbidden violence and the code of forbidden knowledge. In these codes are woven together both mythical themes and human experiences." (KVANVIG 2007, S. 156)

52 Vgl. Nickelsburg 1977, S. 385. 
lung verbunden. Kampf und Strafgericht an den Wächtern sind uns in dem sogdischen Gigantenbuch-Fragment G teilweise überliefert. ${ }^{53}$ Wie die Menschen (und die überbevölkerte Erde) himmlischer Intervention bedürfen, um die Gewalt der Giganten zu beenden, so bedarf der Manichäer des Eingreifens des Licht-Nous, um den geistigen Transformationsprozess $\mathrm{zu}$ bewerkstelligen. Deutlich wird im 38. Kephalaion gesagt:

„Wie in den Wachbezirken seiner großen Brüder, die sich außen befinden, die

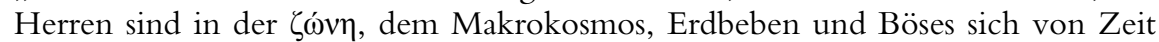
zu Zeit ereignet haben, so geschah es auch im Wachbezirk des Nus, d.i. der Fleischeskörper: die Sünde erweckt von Zeit zu Zeit ihre Verwirrungen im Körper." (Kephalaia Kap. 38, S. 94.22-27) ${ }^{54}$

Die mit der Bestrafung und Fesselung der Wächter beauftragten Engel der Henochtradition und somit auch des manichäischen Gigantenbuches auf narrativer Ebene haben ihr funktionales Analogon in der Gestalt des Licht-Nous, der die negativen Affekte ,,bindet“. Zu vergleichen ist auch das 70. Kephalaion, das dezidiert Makro- und Mikrokosmos miteinander in Verbindung setzt:

„,Wie] diese fünf Wachen sich in dieser großen [Fessel? dieser fünf] Lager $(\pi \alpha \rho \varepsilon \mu \beta o \lambda \eta ́)$ befinden, über die ich zu euch gesprochen habe, so steht es auch mit diesem Körper, den die Electi anlegen ( gibt es in ihm, die der Licht-Noû ̧ bewacht sowie der Neue Mensch, der mit ihm ist." (Kephalaia Kap. 70, S. 171.28-172.4)

Wer über sein Gesicht, Herz, Schoß, Magen und Füße Herr ist, d.h. die hierauf bezogenen falschen Handlungen unterlässt, gleicht den fünf Söhnen des Lebendigen Geistes (ebd., S. 172.4-20). Die makro-mikrokosmischen Äquivalenzen werden also weiter entwickelt. Die kosmischen Rebellionen werden in Beziehung gesetzt zur Rebellion auf anthropologischer Ebene. Hiermit ergibt sich die Einbindung des Gigantenbuchstoffes in die manichäische Lehre in Form einer Makrokosmos-Mikrokosmos-Spekulation, die ausführlich im 70. Kephalaion behandelt wird. ${ }^{55}$

Der Sermon vom Licht-Nous hat vermutlich seine Wurzeln in der allegorischen Auslegung des Gigantenbuches am Ende dieses Werkes, wie sie sich uns in dem von SUNDERMANN edierten mittelpersischen traktatartigen Text der Handschrift S I O/120 (= L) präsentiert. Er ist also von seinem Ursprung her eine Art erweiterter Kommentar zum Gigantenbuch. ${ }^{56}$ Hierauf könnte auch der

53 Siehe HeNNING 1943-1946, S. 68-69 [131-132]. Auch wird dort davon berichtet, dass die 200 Wächter sich vor den strafenden Engeln fürchteten und sich unter den Menschen versteckten. Die Engel trieben sie mit Gewalt auseinander und setzten Wächter über sie.

54 Leicht modifizierte Übersetzung in BÖHLIG/ASMUSSEN 1995, S. 181.

55 S. $169.23-175.24$.

56 So würde sich auch erklären, warum beide Werke in einer alttürkischen Handschrift gemeinsam überliefert worden sind, denn ihr originärer Zusammenhang war den Manichäern Zentralasiens noch bewusst. Zu den dogmatischen Unterschieden zwischen 
literarische Terminus parthisch wifrās im Titel Manohmed rō̌sn wifrās (= Sermon vom Licht-Nous) hindeuten, da dieser auch die Bedeutung 'Kommentar' tragen kann. ${ }^{57}$ Das Gigantenbuch bedurfte aus folgendem Grunde einer allegorischen Auslegung durch die Manichäer: die Vorstellungen von der Herkunft des Bösen waren in der Henochliteratur und bei Mani grundsätzlich verschieden. Leitete die Henochliteratur den Ursprung des Bösen aus einer himmlischen Rebellion ab, bedeutete also eine Art „Sündenfall“58, so war nach Mani das Böse von Ewigkeit her bereits existent als ein dem Guten diametral entgegengesetztes Prinzip. ${ }^{59}$ Die von ihm konzipierten religiösen Funktionsgestalten ließen die traditionelle jüdisch-apokalyptische Interpretation nicht $\mathrm{zu} .{ }^{60}$ Auch ist die Art der Vermischung von himmlischen Wesen mit den Menschen in Manis Theologie nicht vorgesehen, da es bei ihm um die Vermischung von Licht und Finsternis geht. Der jüdische Mythos bezieht sich auf eine unerlaubte Annäherung der himmlischen Sphäre (Wächter) und der irdischen Sphäre (Töchter der Menschen), die zu einer Befleckung der geistigen Natur der Wächterengel führt. ${ }^{61}$ Die Dichotomie heilig vs. profan, die für den jüdischen Mythos als konstitutiv betrachtet werden kann, ${ }^{62}$ kann nicht für den manichäischen Dualismus in Anspruch genommen werden. Die Oppositionen sind also grundsätzlich andere. Aber auch bei Mani geht es letzten Endes um Scheidung und Entmischung, und in der „Deutung“ des Gigantenbuchs wird dies explizit in anthropologisch-soteriologischer Begrifflichkeit vorgeführt. ${ }^{63}$ Eine wörtliche Lesart des Gigantenbuches hätte sich aber mit der Grundkonzeption der manichäischen Lehre nicht vertragen. Somit wurde bei Mani der Mythos von der Rebellion der Wächter und von deren Verbindung mit den Menschentöchtern

dem Gigantenbuchfragment S I O/120 (= L) und dem Sermon vgl. Sundermann 1992, S. 16.

57 Vgl. hierzu den Beitrag von DeSMOND DURKIN-MEISTERERnST in diesem Band.

58 Zur Frage nach der Auffassung der Sünde in der Henochtradition vgl. BeDENBENDER 2000, S. 192-200.

59 BocCACCINI (2007, S. 319) hat gezeigt, dass die Gemeinde von Qumran, je dualistischer ihr Weltbild wurde, desto mehr ihr Interesse an den Lehren der Henochliteratur verlor. BOCCACCINI nimmt eine Art Schisma zwischen dem henochischen und dem qumranischen Judentum an (loc. cit.). Speziell zur Angelologie der Qumran-Texte siehe DAVIDSON 1992.

60 Dies zeigt sich auch an der Terminologie, mit der die Wächter im manichäischen Gigantenbuch bezeichnet werden: sie gelten als Dämonen. Allerdings begegnet in der mittelpersischen Version auch der aramäische Terminus ìr (' $y r$ ' 'Wächter'.

61 Vgl. REEVES 1992, S. 69.

62 So richtig ReEves 1992, S. 68.

63 Neben der aus dem Sermon vom Licht-Nous bekannten Aussonderung und Bindung der negativen Affekte heißt es wohl vom Licht-Nous: „Er erschuf aus der eigenen Erschaffung die Liebe - Freude, Gnade, ${ }^{*}$ Weite und $\star$ Stille, derart, daß die Liebe selbst der wachsame Wächter sei zwischen den Reinen und den Vermischten." SUNDERMANN 1984, S. 503 [627]. Hier wird die mikrokosmische Ebene ausgeweitet auf die sozioreligiöse Sphäre. 
und die Exzesse der Giganten teilweise entmythologisiert und in die Anthropologie überführt. Die traditionelle Narration wird somit auf kreative Weise dekonstruiert. Das kosmische Drama ist ein Bild für die Anfechtungen, mit denen der Electus als Neuer Mensch zu kämpfen hat. Vorgänge in der Urzeit - nach jüdischer Tradition am vierten Schöpfungstag bzw. zur Zeit Jareds ${ }^{64}$ stattfindend - sind Gleichnis für Prozesse, die im Menschen immer wieder ablaufen. Rebellion, Bindung und Gericht sind auf anthropologischer Ebene ständig zu beobachten. Mit der Parallelisierung der Rebellionen auf makround auf mikrokosmischer Ebene ist eine Brücke zwischen Gigantenbuch und Sermon vom Licht-Nous zu schlagen. Die prinzipiell durch die verschiedenen Funktionsgestalten der Zweiten Berufung gewährleistete Eindämmung von Chaos und Unordnung auf makrokosmischer Ebene gibt in der manichäischen Anthropologie das Modell ab für die Zügelung niederer Triebe und Affektkontrolle auf mikrokosmischer Ebene, nämlich beim Neuen Menschen. Auf makrokosmischer Ebene erfüllen insbesondere die fünf Söhne des Lebendigen Geistes eine wichtige Funktion hinsichtlich der Bezwingung der Dämonen. Im Manichäismus sind Kosmologisches und Anthropologisches von gleicher Relevanz, und die Kenntnis der Vorgänge auf beiden Ebenen ist gleichermaßen unabdingbar für die Erlösung. Insofern ist auch der aus der Gigantenbuchexegese hervorgegangene Sermon vom Licht-Nous nicht nur eine Lehrschrift für Electi, sondern auch über die Electi. Bezeichnenderweise ist diesem Werk eine echt manichäische Kosmogonie vorangestellt ( $\mathrm{b}$ \ 2 der parthischen Fassung $)^{65}$, die aber erst mit der Zeit der Vermischung in dem Moment einsetzt, als der Lebendige Geist und die „Mutter der Gerechten“ mit dem Urmenschen wieder aus der Finsternis in Richtung Licht emporsteigen. Eine weitere Verknüpfung mit dem Mythos von den Giganten ist im Falle des türkischen Sermons vom Licht-Nous darin zu sehen, dass u.a. bei der Beschreibung der kosmogonischen Funktion der Mutter des Lebens neben anderen dämonischen Gestalten auch die bädüklär 'die Riesen' erwähnt werden. ${ }^{66}$

\section{VII.}

Durch die partielle Entmythologisierung der Erzählung vom Fall der Wächter sind Mani und seine Schüler somit nicht nur Überlieferer der jüdischen Apokalyptik, sondern gleichzeitig deren Überwinder. Durch die von Mani angebotene „Deutung“" können die apokalyptischen Visionen durch eine gänzlich neue Perspektive aktuelle Relevanz erhalten. Während die Henochtradition sehr wohl eine Typologie Urzeit-Endzeit kennt und somit die Mythen der Zeit

64 Siehe DelCOR 1976 [1979], S. 23 [283], 25 [285] f.

65 In der Ausgabe von SUNDERMANN 1992.

66 Vgl. WiLKENS 2001/2002, S. 87, 89, 90. 
vor der Sintflut auf das Weltende projiziert, ${ }^{67}$ gibt es im Manichäismus kaum Hinweise darauf, dass der Mythos vom Fall der Engel und ihrer Bestrafung eschatologische Relevanz hat. Allerdings könnte die Einkerkerung der Wächter (Gigantenbuchfragment $\mathrm{G}=\mathrm{M}$ 7800) darauf hindeuten, dass erst am Weltende über sie Gericht gehalten wird. ${ }^{68}$

Die zu Recht erkannte enge Verwandtschaft von Gigantenbuch und Sermon vom Licht-Nous ist die literarhistorische Basis, um inhaltliche Vergleiche zwischen beiden Werken anzustellen. Eine mögliche Bezugnahme auf psychologische Themen des Gigantenbuchs könnte $\int 84$ des Sermons darstellen, in dem die „zwölf finsteren Herrschaften“ aufgezählt werden, die negativen Entsprechungen zu den zwölf Lichtherrschertümern:

„Die zwölf finsteren Herrschaften. Die erste ist die Torheit, die zweite ist die Begehrlichkeit, die dritte ist die Selbstüberhebung, die vierte ist der Aufruhr, die fünfte ist der Z[orn], die sechste ist die Befleckung, [die sie]bente ist die Zerstörung, die achte ist das [Ver]derben, die neunte ist der Tod, die zehnte ist der Trug, die elf[te] ist die Rebellion, die zwölfte ist die Finsternis." (SUNDERMANN 1992, S. 73)

SUNDERMANN bemerkt in seinem Kommentar zur Stelle ${ }^{69}$ zu Recht, dass diese zwölf Herrschaften der Finsternis keine „mechanische[n] Nachbildungen der lichten sein (können)“, sondern sie gäben ,einem anderen, noch unbekannten Gedanken Ausdruck“. Eventuell wird diese Reihe aus dem Gigantenbuch gespeist, da sie alle sehr gut das Verhalten der Wächter und der Giganten und die damit zusammenhängenden Auswirkungen charakterisieren. Die „zwölf finsteren Herrschaften“ muten wie Leitmotive des Gigantenbuchs an. Sie könnten somit auf eine Lehrtradition zurückgehen, die auf das Gigantenbuch Bezug nimmt. Nur die Finsternis dürfte nicht hierzu gehören, aber dieser Begriff ist auch der einzige, der ein Gegenstück in der Reihe der zwölf Lichtherrschertümer hat.

\section{VIII.}

Mani hat mit seiner Deutung des Gigantenbuches neue Wege in der spätantiken Exegese aufgewiesen. Er unterscheidet sich hierin von der häufiger belegten „Protestexegese“ gnostischer Zirkel, die z. B. mythische Erzählungen der hebräischen Bibel in ihrem Sinne auslegten, aber dem Mythischen verhaftet blieben. Mani hingegen konnte die Verbindung alter, wenn auch leicht modifizierter Mythen mit psychologisch-anthropologischen Konzepten verbinden.

67 Vgl. Nickelsburg 1977, S. 388 und Hanson 1977, S. 197.

68 Eine solche Sichtweise ist bereits im Wächterbuch, besonders in den Kapiteln 18-19, angelegt. Siehe Collins 1998, S. 58.

69 1992, S. 118. 
Erneut zeigt sich hierin die Originalität von Manis Theologie. ${ }^{70}$ Interessant scheint mir die Tatsache zu sein, dass Alexander von Lykopolis in seiner antimanichäischen Schrift, die in etwa um das Jahr 300 n. Chr. entstanden sein dürfte, davon spricht, dass die Dichter den Mythos von den Engeln, die mit den Töchtern der Menschen verkehren, und dem Gigantengeschlecht in allegorischer Weise verwendeten und auf die Seele und den Körper bezögen. ${ }^{71}$ Die Manichäer verständen von all dem jedoch nichts. ${ }^{72}$ Wie das mittelpersische Gigantenbuchblatt S I O/120 (= L) zeigt, verstand Mani sehr wohl etwas davon.

Nun war Mani nicht der einzige spätantike Autor, der den Mythos vom Fall der Engel mit einer spezifischen Psychologie verband. Auch der philosophisch gebildete christliche Autor Athenagoras von Athen entwickelt in seinem Bittgesuch an Marcus Aurelius, der Legatio pro christianis (25,1-3), die in den 170er Jahren entstanden ist, eine interessante psychologische Interpretation. ${ }^{73}$ In seiner Auslegung des Mythos von der Rebellion der Wächter rekurriert Athenagoras auf einen Dualismus Prinz (پ̋ $\rho \omega \omega)$ oder Geist $(\pi v \varepsilon \tilde{v} \mu \alpha)$ der Materie vs. reiner bzw. himmlischer Geist. ${ }^{74}$ Dämonen können ihm zufolge in den menschlichen Seelen Bewegungen und Aktivitäten hervorrufen, und zwar in den Einzelseelen oder auch bei ganzen Völkern. ${ }^{75}$ Die bösen Affekte haben ihr Gegenstück in der Kontemplation des Göttlichen. ${ }^{76}$ Ohne hier historische Bezüge herstellen zu wollen, so zeigt dieses Beispiel, dass der Mythos vom Fall der Wächter im christlich-hellenistischen Milieu nicht allein wörtlich genommen, sondern wie bei Mani zugleich auch psychologisch gedeutet werden konnte. ${ }^{77}$

70 Mit seiner Deutung des Gigantenbuches hat der Religionsstifter sicher den Anspruch verbunden, die Authentizität der richtigen Lehre wiederherzustellen. An diesen Themenkreis ließen sich weitere Fragen anknüpfen, z. B. ob Mani seine in der „Deutung“ dargelegten Lehren erst anhand der Mythen des Gigantenbuches entwickelt hat oder ob er jene schon vorher ausgearbeitet und sekundär dem Text des Gigantenbuches nachgeschaltet hat. Ohne hier eine abschließende Antwort geben zu können, scheint mir die zweite Alternative wahrscheinlicher zu sein.

71 Eine solche allegorische Auslegung vertrat auch Philo von Alexandrien in seiner Schrift Über die Giganten. Proclus interpretierte die Gigantomachie der antiken Tradition als Kampf zwischen der Vernunft und den niederen Leidenschaften. Vgl. Mussies 1999, S. 343b.

72 Die Stelle bei ReEves (1992, S. 21) in Übersetzung.

73 GIULEA 2007.

74 Op. cit., S. 268.

75 Op. cit., S. 270

76 Op. cit., S. 273.

77 „Finally, from the prism of theological language, Athenagoras's contribution might represent a link not only between two emblematic texts, but also between two different ways of articulating a theological discourse: from a (more) mythological (Jewish, in this instance) one to the Greek and (more) philosophical-conceptual one." (op. cit., S. 281). 
Eine literar- und gattungsgeschichtliche Frage schließt sich an: Wenn das Schema eines Textes, der durch eine davon getrennte allegorische Ausdeutung erklärt wird, bereits im Gigantenbuch präsent ist, was sagt das aus über die Parabelliteratur, deren Ursprünge meist im Osten der manichäischen Welt gesucht wurden? Zumindest lässt sich sagen, dass die bereits aus einem kanonischen Werk Manis bekannte literarische Form die Adaption kürzerer Formen (Erzählungen mit Epimythion) begünstigt haben muss. Wie das Gigantenbuch ohne die spezifisch manichäische Deutung nicht vollständig ist, so sind auch die Parabeln ohne ihre Epimythien unvollständig. Die narrativen Texte weisen über sich hinaus. Der reine Wortsinn ist etwas Vorläufiges, das erst erläutert werden muss. Und nur durch die Erläuterung werden die Texte überhaupt zu manichäischen Texten. Hiermit kommt es im Falle des Gigantenbuchs auch zu einem Genrewechsel. Eine apokalyptische Urgeschichtserzählung gerät zur Parabel. ${ }^{78}$ Wenn das Gigantenbuch speziell in der Ostmission von Bedeutung war, dann sicherlich gerade auch die Deutung der Vorgänge am Ende des Buches, die signalisieren, wie man nach manichäischem Verständnis zur epischen Tradition Stellung zu nehmen hat. ${ }^{79}$ Seine Exegese postuliert eine typologische Äquivalenz von kosmischer und anthropologischer Ebene. Die Traditionen, die uns in den Kephalaia begegnen, in denen die Rebellion im Wachbezirk des

78 Ähnlich auch SUNDERMANN in Bezug auf das 38. Kephalaion: „Was aber das Kephalaion so sehr hervorhebt, die Rebellion der Dämonen im Makrokosmos und die Rebellion des Alten Menschen im Mikrokosmos, das paßt in geradezu idealer Weise zu Manis Gigantenbuch. Nicht nur wird im Kephalaion der uns bekannte mythologische Gegenstand des Gigantenbuches exzerpierend nacherzählt (S. 93,23-28), das Kephalaion dürfte auch eine Antwort auf die Frage liefern, warum, mit welcher didaktischen $\mathrm{Ab}$ sicht Mani die absonderlichen Mythen von urzeitlichen ,Wächtern' und ihren gigantischen Söhnen nacherzählte. [...] Sie wurden, wie jede Erzählung, die Mani und die Seinen schufen, zur Parabel." (SUNDERMANN 1992, S. 15). Zum parabelhaften Charakter des Gigantenbuchs s. auch SUNDERMANN 2009, S. 216.

79 Eine Schlussfolgerung drängt sich auf der Basis des oben Gesagten auf: Wie Henoch als Patriarch bzw. im manichäischen System als Lichtapostel der Vorzeit über das Wissen verfügt, die verschlüsselten Träume der Giganten zu deuten und auf das bevorstehende kosmische Geschehen zu beziehen, so ist Mani in der Lage, den narrativ vermittelten Stoff des Gigantenbuchs psychologisch zu deuten. Ein Unterschied trotz der zu beobachtenden typologischen Äquivalenz ist hinsichtlich der Adressaten festzustellen: Während im Falle Henochs die Giganten ihrem Schicksal nicht entrinnen können und ihnen ihr Ende angekündigt wird, wendet sich Mani an die Electi, um sie vor drohenden Rebellionen in ihrem eigenen Selbst nach der Umgestaltung zum Neuen Menschen zu warnen. Der Prozess der Konversion ist reversibel und daher bedürfen die Electi einer fortgesetzten Selbsterforschung. HutTER (2004, S. 135b) bezieht auch die Spuren der Parabeln im manichäischen Gigantenbuch in diesen Zusammenhang ein: „Dem Menschen, der in diesem Kampf selbst mitwirken muß, wird dabei durch die unterschiedlichen Parabeln zugleich ein ethisches Verhalten vor Augen gestellt, das ihn für den Kampf auf der Seite des Lichtes motivieren soll.“ 
Rex Honoris lokalisiert wird, zeigen aber, dass den Manichäern daran gelegen war, das mythische Element zu bewahren und nicht zur Gänze zu allegorisieren.

\begin{tabular}{|c|c|}
\hline \multicolumn{2}{|r|}{ Abkürzungen } \\
\hline BSOAS & Bulletin of the School of Oriental and African Studies \\
\hline DDD & KAREL VAN DER TOORN, BOB BECKING und PIETER W. VAN \\
\hline & DER HORST (Hrsg.), Dictionary of Deities and Demons in the Bible. \\
\hline & Leiden u.a. 1999. Second Extensively Revised Edition. \\
\hline $\mathrm{L}$ & Journal of Biblical Literature \\
\hline & Journal for the Study of Judaism \\
\hline AWG & $\begin{array}{l}\text { Nachrichten der Akademie der Wissenschaften in Göttingen, I. } \\
\text { Philologisch-Historische Klasse }\end{array}$ \\
\hline AW & Sitzungsberichte der Preußischen Akademie der Wissenschaften \\
\hline DMG & Zeitschrift der Deutschen Morgenländischen Gesellschaft \\
\hline RGG & Zeitschrift für Religions- und Geistesgeschichte \\
\hline
\end{tabular}

Bibliographie

ASSMANn, JAN ( $\left.{ }^{4} 2002\right):$ Das kulturelle Gedächtnis. Schrift, Erinnerung und politische Identität in frühen Hochkulturen, München.

Auffarth, Christoph / Loren T. Stuckenbruck (Hrsg.) (2004): The Fall of the Angels, Leiden u. a. (Themes in Biblical Narrative; Jewish and Christian Traditions 6).

BAuckHAm, Richard J. (1985): The Fall of the Angels as the Source of Philosophy in Hermias and Clement of Alexandria. In: Vigiliae Christianae 39, S. 313-330.

BeDEnBEnder, AndReAs (2000): Der Gott der Welt tritt auf den Sinai. Entstehung, Entwicklung und Funktionsweise der frühjüdischen Apokalyptik, Berlin (Arbeiten zur neutestamentlichen Theologie und Zeitgeschichte 8).

BoCCACCINI, GABRIELE (2007): Enochians, Urban Essenes, Qumranites: Three Social Groups, One Intellectual Movement. In: BocCACCINI/COLlins 2007, S. 301327.

Boccaccini, Gabriele / John C. Collins (Hrsg.) (2007): The Early Enoch Literature, Leiden/Boston (Supplements to the Journal for the Study of Judaism 121).

Böhlig, Alexander / Jes Peter Amussen (21995): Die Gnosis: Der Manichäismus. Unter Mitwirkung von Jes Peter Asmussen eingeleitet, übersetzt und erläutert von Alexander Böhlig, Zürich.

Collins, John J. (21998): The Apocalyptic Imagination: An Introduction to Jewish Apocalyptic Literature, Grand Rapids, Mich. u.a.

Collins, John J. (2007): How Distinctive was Enochic Judaism? In: Meghillot 5-6, S. 17-34.

Coxon, P. W. (1999): [Artikel] Nephilim. In: DDD, S. 618b-620b. 
Davidson, Maxwell J. (1992): Angels at Qumran: A Comparative Study of 1 Enoch 136, 72-108 and Sectarian Writings from Qumran, Sheffield (JSP Supplement Series 11).

DelCOR, MATHIAS (1979): Le mythe de la chute des anges et de l'origine des géants comme explication du mal dans le monde dans l'apocalyptique juive. Histoire des traditions. In: Revue de l'Histoire des Religions 189 (1976), S. 3-53 [Nachdruck in: DERS., Études bibliques et orientales de religions compares, Leiden, S. 263-313].

Durkin-Meisterernst, Desmond / Enrico Morano (2010): Mani's Psalms. Middle Persian, Parthian and Sogdian fragments in the Turfan Collection, Turnhout (Berliner Turfantexte XXVII).

FunK, Wolf-Peter (2009): Manis Account of Other Religions According to the Coptic Synaxeis Codex. In: JASON DAvid BeDuHn (Hrsg.), New Light on Manichaeism. Papers from the Sixth International Congress on Manichaeism Organized by the International Association of Manichaean Studies, Leiden/Boston (Nag Hammadi and Manichaean Studies 64), S. 115-127.

GARDNer, IAIN / SAmUel N. C. LIEU (2004): Manichaean Texts from the Roman Empire, Cambridge.

GiUleA, Dragoş-Andrei (2007): The Watchers' Whispers: Athenagoras's Legatio 25,1-3 and the Book of the Watchers. In: Vigiliae Christianae 61, S. 258-281.

Hanson, Paul D. (1977): Rebellion in Heaven, Azazel, and Euhemeristic Heroes in 1 Enoch 6-11. In: JBL 96, S. 195-233.

Heger, Paul (2010): 1 Enoch - Complementary or Alternative to Mosaic Torah? In: JSJ 41, S. 29-62.

Hendel, Ronald S. (1987): Of Demigods and the Deluge: Toward an Interpretation of Genesis 6:1-4. In: JBL 106, S. 13-26.

HeNNING, W. B. (1934): Ein manichäisches Henochbuch, Berlin 1934. SPAW, S. 27-35 [Nachdruck in: Selected Papers I, S. 341-349].

HeNNING, W. B. (1943-1946): The Book of Giants. In: BSOAS XI, S. 52-74 [Nachdruck in: Selected Papers II, S. 115-137].

HutTer, MANFRED (1992): Manis kosmogonische Šābuhragān-Texte. Edition, Kommentar und literaturgeschichtliche Einordnung der manichäisch-mittelpersischen Handschriften $M$ 98/99 I und M 7980-7984, Wiesbaden (Studies in Oriental Religions 21).

HutTer, MANFRed (2004): Zur Funktion des Beginns von Manis Gigantenbuch. In: Desmond Durkin-Meisterernst et al. (Hrsg.), Turfan Revisited. The First Century of Research into the Arts and Cultures of the Silk Road, Berlin (Monographien zur Indischen Archäologie, Kunst und Philologie 17), S. 134a-137a.

Jackson, David R. (2004): Enochic Judaism. Three Defining Paradigm Exemplars, London/New York (Library of Second Temple Studies 49).

KlimkeIT, HANS-JOACHIM (1980): Der Buddha Henoch: Qumran und Turfan. In: ZRGG 32, S. 367-377.

KniBB, Michael A. (2001): Christian Adoption and Transmission of Jewish Pseudepigrapha: The Case of 1 Enoch. In: JSJ 32, S. 396-415.

KNiBB, Michael A. (2007): The Book of Enoch or Books of Enoch? The Textual Evidence for 1 Enoch. In: BocCACCINI/COLLINS 2007, S. 21-40. 
Kvanvig, Helge S. (1988): Roots of Apocalyptic. The Mesopotamian Background of the Enoch Figure and the Son of Man, Neukirchen-Vluyn (Wissenschaftliche Monographien zum Alten und Neuen Testament 61).

Kvanvig, Helge S. (2007): Cosmic Laws and Cosmic Imbalance: Wisdom, Myth and Apocalyptic in Early Enochic Writings. In: BocCACCINI/COLLINS 2007, S. 139158.

MaCKenZIE, DAVID NeIL (1979): Mani’s Šābuhragān I. In: BSOAS 42, S. 500-534.

MaCKenZIE, DAVID NeIL (1980): Mani’s Šābuhragān II. In: BSOAS 43, S. 288-310.

MiLik, J. T. (1971): Turfan et Qumran. Livre des Géants juif et manichéen. In: GeRT Jeremias / Heinz-Wolfgang Kuhn / Hartmut Stegemann (Hrsg.), Tradition und Glaube: Festgabe K. G. Kuhn zum 65. Geburtstag, Göttingen, S. 117-127.

Milik, J. T. (1976): The Books of Enoch. Aramaic Fragments of Qumrân Cave 4, Oxford.

Morano, ENRico (2008): Il «libro dei Giganti» di Mani. In: GHERARdo GNOLI (Hrsg.), Il Manicheismo. Volume III: Il mito e la dottrina. Testi manichei dell'Asia Centrale e della Cina, [Milano], S. 69-107.

MORANO, ENRICO (2009): "If they had lived ..." A Sogdian-Parthian Fragment of Mani's Book of Giants. In: Werner SUndermanN / ALMUt Hintze / FranÇOIS DE BloIS (Hrsg.), Exegisti Monumenta. Festschrift in Honour of Nicholas Sims-Williams, Wiesbaden (Iranica 17), S. 325-330.

MussiES, G. (1999): [Artikel] Giants. In: DDD, S. 343a-345a.

Nickelsburg, George W. E. (1977): Apocalyptic and Myth in 1 Enoch 6-11. In: JBL 96, S. 383-405.

Nickelsburg, George W. E. (2001): 1 Enoch 1: A Commentary, Hermeneia/Minneapolis.

Perlitt, Lothar (1990): Riesen im Alten Testament, Göttingen (NAWG 1990, Nr. $1)$.

[Polotsky, Hans JaKoB / Alexander BÖHlig] (1940): Kephalaia. 1. Hälfte (Lieferung 1-10). Mit einem Beitrag von HUGO IBSCHER, Stuttgart (Manichäische Handschriften der Staatlichen Museen Berlin 1).

PueCh, Émile (2000): Les songes des fils de Šemiḥazah dans le Livre des Géants de Qumrân. In: Académie des inscriptions et belles-lettres, 144e année. N. 1, S. 7-26.

ReCK, ChRistiane (2006): Mitteliranische Handschriften Teil 1: Berliner Turfanfragmente manichäischen Inhalts in soghdischer Schrift, Stuttgart (Verzeichnis der Orientalischen Handschriften in Deutschland XVIII, 1).

Reck, Christiane / Dieter Weber (Hrsg.) (2001): Manichaica Iranica: Ausgewählte Schriften von Werner Sundermann. Herausgegeben von CHRISTIANE RECK und DieTer WeBer unter Mitarbeit von Claudia LeUrini und ANTONio PANAINO. Bd. 1-2, Roma (Serie Orientale Roma LXXXV, 1-2).

Reed, AnNette Yoshiko (2005): Fallen Angels and the History of Judaism and Christianity: The Reception of Enochic Literature, Cambridge.

Reeves, J. C. (1991): An Enochic Motif in Manichaean Tradition. In: Aloïs VAN Tongerloo und Søren Giversen (Hrsg.), Manichaica Selecta. Studies Presented to Professor Julien Ries on the Occasion of his Seventieth Birthday, Louvain (Manichaean Studies I), S. 295-298. 
ReEves, J. C. (1992): Jewish Lore in Manichaean Cosmogony. Studies in the Book of Giants Traditions, Cincinnati (Monographs of the Hebrew Union College 14).

REEVES, J. C. (1993): Utnapishtim in the Book of Giants? In: JBL 112, S. 110-115.

REEves, J. C. (1996): Heralds of That Good Realm: Syro-Mesopotamian Gnosis and Jewish Traditions, Leiden/New York/Köln (Nag Hammadi and Manichaean Studies 41).

SkJÆRvø, Prods OKTOR (1995): Iranian Epic and the Manichean Book of Giants. Irano-Manichaica III. In: Acta Orientalia Academiae Scientiarum Hungaricae XLVIII.1-2, S. 187-223.

STUCKenbruck, LOREN T. (1997a): The Book of Giants from Qumran, Tübingen (Texte und Studien zum Antiken Judentum 63).

StuCKenBRUCK, LOREN T. (1997b): The Sequencing of Fragments Belonging to the Qumran Book of Giants: An Inquiry into the Structure and Purpose of an Early Jewish Composition. In: Journal for the Study of the Pseudepigrapha 16, S. 3-24.

StuCKenbruck, Loren T. (2003): Giant Mythology and Demonology: From the Ancient Near East to the Dead Sea Scrolls. In: Armin LANGe / Hermann LiCHTenberger / K. F. Diethard Römheld (Hrsg.), Die Dämonen / Demons: Die Dämonologie der israelitisch-jüdischen und frühchristlichen Literatur im Kontext ihrer Umwelt / The Demonology of Israelite-Jewish and Early Christian Literature in Context of their Environment, Tübingen, S. 318-338.

STUCKEnBRUCK, LOREN T. (2007): The Early Traditions Related to 1 Enoch from the Dead Sea Scrolls: an Overview and Assessment. In: BocCACCINI/COLLINS 2007, S. 41-63.

SUNDERMANN, WeRnER (1984): Ein weiteres Fragment aus Manis Gigantenbuch. In: Orientalia J. Duchesne-Guillemin emerito oblata, Leiden, S. 491-505 [Nachdruck in: RECK/WEBER 2001, Bd. 2, S. 615-631; die russische Version mit einem Faksimile erschien unter dem Titel Ešče odin fragment iz „knigi gigantov“ Mani. In: Vestnik Drevnej Istorii 190 (1989), S. 67-79].

SundermanN, Werner (1992): Der Sermon vom Licht-Nous. Eine Lehrschrift des östlichen Manichäismus. Edition der parthischen und sogdischen Version, Berlin (Berliner Turfantexte XVII).

SUNDERMANN, Werner (1994a): Mani’s „Book of the Giants“ and the Jewish Books of Enoch. A Case of Terminological Difference and What it Implies. In: SHAUL Shaked / Amnon Netzer (Hrsg.), Irano-Judaica III, Jerusalem, S. 40-48 [Nachdruck in: RECK/WEBER 2001, Bd. 2, S. 697-706].

SundermanN, Werner (1994b): Iranische Personennamen der Manichäer. In: Die Sprache 36/2, S. 244-269 [Nachdruck in: RECK/WEBER 2001, Bd. 1, S. 485-513].

SUNDERMANN, WERNER (2009): Manichaean Literature in Iranian Languages. In: RONALD E. EMMERICK / MARIA MACUCH (Hrsg.), The Literature of Pre-Islamic Iran. Companion Volume I to A History of Persian Literature, London/New York (A History of Persian Literature XVII), S. 197-265.

VAnderKam, James C. (2007): Mapping Second Temple Judaism. In: BocCACCINI/COLLINS 2007, S. 1-20.

Wilkens, Jens (2000): Neue Fragmente aus Manis Gigantenbuch. In: ZDMG 150, S. 133-176. 
WiLkENS, JENS (2001/2002): Der manichäische Traktat in seiner alttürkischen Fassung - neues Material, neue Perspektiven. In: Ural-Altaische Jahrbücher Neue Folge 17, S. $78-105$.

Witakowski, Witold (2009): The Fall of Angels in Ethiopian Tradition. In: Rocznik Orientalistyczny LXII.1, S. 240-249.

ZimmermanN, Mirjam / Ruben ZimmermanN (1999): ,Heilige Hochzeit' der Göttersöhne und Menschentöchter? Spuren des Mythos in Gen 6,1-4. In: Zeitschrift für die alttestamentliche Wissenschaft 111, S. 327-352. 
